\title{
Do managers of South African manufacturing firms make optimal capital investment decisions?
}

\author{
E. Gilbert \\ Graduate School of Business, University of Cape Town \\ Private Bag, Rondebosch 7701, Republic of South Africa \\ gilberte@gsb.uct.ac.za
}

Received May 2003

\begin{abstract}
Managing the deployment of a firm's assets in the form of capital goods is a vital determinant of a firm's ability to successfully compete over time. Corporate finance theory clearly prescribes a rule to ensure the optimality of these decisions: all capital investment decisions should be evaluated through the use of the net present value (NPV) rule while project specific risk should be incorporated through the adjustment of the discount rate used in the NPV analysis.

This paper presents the results of a survey of the capital investment evaluation practices of South African manufacturing firms. The results indicate that the majority of firms surveyed do not use the NPV evaluation technique when making their capital investment decisions. Furthermore, those firms that do use this technique use it in combination with other, theoretically deficient (and redundant) techniques. Finally, they do not adjust for project specific risk as prescribed.
\end{abstract}

\section{Introduction}

Correct capital investment decisions are vital to the ability of firms to continue to produce and compete successfully. However, they represent a risk to the firm since they are normally costly to reverse. Judging whether or not to invest in additional or newer versions of capital at any point in time is thus a key managerial decision.

With respect to these decisions, the prescriptive conclusion of traditional corporate finance theory is that a positive net present value (NPV) criterion (using a discount rate appropriately adjusted for risk) is the optimal capital investment evaluation method ${ }^{1)}$. Alternative techniques of evaluating capital investments exist but are deficient in terms of achieving the goal of maximising the firm's net present value. Furthermore, their use does not add to the decision maker's ability to achieve this goal. There is no (theoretical) reason for their use.

In this paper the results of a survey of the capital budgeting practices in the manufacturing sector in South Africa are reported. This survey was designed to test the degree of congruence between these theoretical prescriptions and manager's behaviour in this regard.

\section{The theory of optimal capital investment decisions}

Capital investment involves the commitment of current resources to projects which will deliver returns in the future.

\footnotetext{
${ }^{1)}$ One alternative approach not commonly addressed by this traditional treatment of capital budgeting is that of Real Options. This approach is not directly addressed in this paper.
}

Assuming that rational individuals will act so as to maximise their utility over time, an optimal investment decision is one which achieves this aim. Given that the exact form of these decisions will depend on the particular tastes of the individuals, that is, by their preference for consumption now as versus consumption later, and that firms are owned by many different shareholders - it would seem impossible to make investment decisions to maximise each separate shareholder's utility ${ }^{2)}$. However, if a perfect capital market exists, that is, one where the rates of borrowing and lending are equal - then it can be shown that the investment decisions of the firm can be taken independently of the consumption decisions of the individual shareholders (Fisher's separation theorem). The opportunities for borrowing and lending offered by perfect capital markets mean that individuals can maximise their consumption choices over time in a way that is unrestricted by other consumers' tastes or by the cash flows of the firm. However, as the shareholders' set of potential consumption alternatives over time is affected by the net present value of the firm, rational shareholders will want the firm to make investment decisions in such a way as to maximise its net present value. The one rule which will always ensure that this aim is achieved is the positive NPV rule. Capital investment projects which have a positive NPV should be selected and those with a negative NPV, rejected. Two of the most important assumptions underlying this prescription are the existence of a perfect capital market and the lack of uncertainty.

\footnotetext{
${ }^{2)}$ One alternative approach not commonly addressed by this traditional treatment of capital budgeting is that of Real Options. This approach is not directly addressed in this paper.
} 
The first assumption is probably the most important as it is required for Fisher's separation theorem to hold. The presence of a perfect capital market allows for the separation of production decisions from consumption decisions. This means that consumers are able to manage their intertemporal consumption decisions so as to maximise their inter-temporal utility independently of the firm's investment and production decisions and the resulting cash flows. Correspondingly firms' decisions are not constrained by the desired consumption patterns of their owners. If the firm's decision makers do not need to take the owners' consumption patterns into account when making its investment decisions, how should these decisions be made? If it is assumed (for convenience) that the owners run the firms, the firm's investment decisions will be carried out so as to maximise the wealth available to the owners. ${ }^{3)}$ This is equivalent to the firms acting so as to maximise their net present value. Thus any optimal rule will require that firms act so as to achieve this aim. If this assumption of the existence of perfect capital markets is relaxed, the firm's decisions will be affected by its owner's consumption pattern desires and the NPV rule will no longer (necessarily) be optimal.

The other important assumption is that the firm's future cash flows are known with certainty. If this is not the case, it is incorrect to assume that firms should necessarily attempt to maximise their expected net present value. ${ }^{4)}$ Thus when dealing with uncertain future cash flows, the Fisher separation theorem does not necessarily hold. However, it can be shown that, using state-preference theory, the Fischer separation condition will still hold under conditions of uncertainty - if the capital market is complete and perfectly competitive or frictionless. ${ }^{5)}$ A consequence of making decisions in a risky environment is that discount rates have to be adjusted for risks which are specific for the purposes of the project. The proponents of the NPV method have often relied on the Capital Asset Pricing Model (CAPM) for estimations of project risk.

Copeland and Weston (1992) highlight four requirements for an optimal technique for the evaluation and thus selection of a capital investment project by a firm. It should include a consideration of all cash flows; these cash flows should be discounted at the opportunity cost of funds; the technique should identify the best project for the firms from a range of mutually exclusive projects; and it should allow managers to consider each project in isolation (the valueadditivity principle).

\footnotetext{
${ }^{3)}$ In practice, however, firms are not run by their owners but rather by professional management. Consequently it is necessary to make the additional assumption that these managers will run the firm with the aim of maximising the owners' potential consumption choices, that is, the net present value of the firm. When the NPV rule is referred to as being optimal it must be realised that it is optimal from the point of view of the owners (and not necessarily the managers). While the validity of this assumption has been questioned it will not be addressed in this paper.

${ }^{4)}$ See Nickell (1978).

${ }^{5)}$ See Copeland and Weston (1992: 125).
}

Of the four most commonly used evaluation techniques (Payback Period (PP), Accounting Rate of Return (ARR), Internal Rate of Return (IRR), and NPV), only the NPV technique meets the four stated requirements. PP and ARR are rejected for their lack of discounting of future cash flows and the fact that the ARR uses accounting profits and not cash flows to value capital investments; NPV is preferred to IRR because the NPV approach gives the exact addition to shareholders' wealth and the IRR approach can result in the correct selection not being made in cases of mutually exclusive projects as it makes a (potentially) incorrect reinvestment rate assumption. The IRR approach implicitly assumes all cash flows are reinvested at the IRR - when the correct measure is the opportunity cost rate (or cost of capital), and also violates the value additivity principle.

The prescriptive conclusion of corporate finance theorists is that the application of a positive NPV criterion (using a discount rate appropriately adjusted for risk) is the optimal approach to evaluating capital investment projects.

\section{Focus of the survey}

The purpose of this study was to capital budgeting behaviour of manufacturing firms in South Africa. There were three reasons for this choice of focus:

1. The manufacturing sector of the South African economy is already an extremely important part of the South African economy. This importance is expected to grow as the government implements policies designed to encourage the restructuring of the South African economy to make it more export orientated, and producing value added goods (i.e. not only commodities).

2. Manufacturing firms are relatively capital intensive. This makes the capital investment decision all that more important in this context.

3. Whilst the work of Andrews and Butler (1986), Parry and Firer (1990) and Hall (2000 \& 2001) include the behaviour of some manufacturing firms in South Africa, no work has been done with an explicit focus on identifying the capital expenditure techniques used by manufacturing firms in South Africa. This study will provide an industry specific data set that will provide a basis for comparison for future research.

\section{Research propositions}

In order to establish the degree of congruency between the behaviour of these firms and the prescriptions of the theory outlined above, the following propositions regarding the capital budgeting behaviour of South African manufacturing firms were identified for further investigation: 


\section{Use of DCF (NPV and IRR) techniques}

1. The majority of manufacturing firms use only the NPV technique when evaluating their capital investment projects (i.e. no other techniques are used at all).

2. All manufacturing firms use at least one DCF technique (NPV and/or IRR) when evaluating their capital investment projects.

3. Of the DCF techniques used, the NPV technique is used more often that the IRR technique.

4. The use of DCF techniques is positively related to manufacturing firm size.

\section{Use of DCF vs. non-DCF techniques}

5. The majority of manufacturing firms do not use nonDCF techniques.

6. Manufacturing firms use DCF techniques more often than they use non-DCF techniques.

\section{Use of combinations of techniques}

7. The manufacturing firms that use DCF techniques use only these techniques (i.e. these firms do not use them in conjunction with other, non-DCF techniques).

8. If a combination of DCF and non-DCF techniques is used, the DCF techniques are applied more often than the non-DCF techniques.

\section{Adjusting for risk}

9. Manufacturing firms adjust the discount rate used in their NPV calculations to incorporate the project specific risk.

\section{Research methodology}

A postal survey of manufacturing firms in South Africa was conducted. The survey aimed to collect information on the relevant facets of the capital expenditure decision from the decision makers themselves, namely: the use of capital expenditure evaluation techniques; and the incorporation of risk through the adjustment of the project discount rate.

An immediate problem was that no sampling frame of this population existed. When approached, Statistics South Africa was unable to help in this regard. However, the South African Chamber of Business (SACOB) offered to support this research. This meant that their list of manufacturing member firms was used as the sample frame.

In August 1997, a pilot version of the questionnaire was circulated to 40 SACOB members selected from those firms which had responded to the most recent monthly Confidence Index survey. Included with the questionnaire were a covering letter and a comments sheet. $\left.{ }^{6}\right)$ There were 24 responses to the pilot questionnaire exercise reflecting a 60 percent response rate. No respondents complained about either the format or the contents of the questionnaire so it was decided to proceed with the main mailing.

Two hundred and eighty two manufacturing companies were targeted in this second survey effort. Of these firms, 22 replied to say that they were either no longer involved with manufacturing activities or were simply agents for foreign manufacturing firms. The surveys sent to two other firms were returned marked 'no longer at this address'. This left an effective sample frame of 258 firms. The 86 usable responses to the main survey thus represent a response rate of 33 percent. These were combined with the 24 responses (out of 60) of the pilot questionnaire giving a total of 110 usable replies. The final response rate was thus 35 percent (110 out of 318 ).

\section{Results}

Table 1 outlines the key characteristics of the respondent firms.

The techniques used by the firms to evaluate their capital expenditure projects are summarised in Table 2. It is clear that DCF techniques (NPV and IRR) are not used most often. When viewed in isolation, the PP and the ROI are, by far, the most popular techniques used by the firms surveyed. Respondents were also asked how often they applied each method they had indicated as being used by their company. The final column of Table 2 indicates what percentage of the time a method is applied in practice. On average, the more popular non-DCF techniques are applied to projects more often than the DCF techniques.

Table 3 presents a summary of how the techniques used vary according to the size of the firm. It suggests that the use of formal techniques by firms in general, as well as that of DCF techniques, is positively related to firm size. Moreover, larger firms tend to apply them more often. The increase in the use of DCF techniques is not mirrored by a decline in the use of non-DCF techniques. In fact, non-DCF techniques are used by a greater number of large firms, and applied to projects even more often. However, the responses for medium firms do cast doubt on the strength of this trend.

Table 4 contains a summary of some of the combinations of techniques used by the firms surveyed. Of the 110 responses, only three firms $(2,8$ percent of the total) use the NPV technique as prescribed by corporate finance theory. Non-DCF techniques dominate the use of DCF techniques. In general, techniques are used in groups, with combination of DCF and non-DCF techniques being the most common grouping.

\footnotetext{
${ }^{6)}$ Dillman's Total Design Method (Dillman, 1978) formed the basis of the design of the content of the covering letter.
} 
Table 1: Details of respondent firms

\begin{tabular}{|c|c|c|c|c|c|}
\hline & 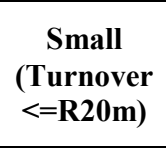 & $\begin{array}{l}\text { Medium } \\
\text { (Turnover } \\
<=\text { R100m) }\end{array}$ & $\begin{array}{c}\text { Large } \\
\text { (Turnover }> \\
\text { R100m) }\end{array}$ & $\begin{array}{c}\text { Other } \\
\text { (no turn-over } \\
\text { data } \\
\text { provided) } \\
\end{array}$ & Total \\
\hline No. of firms & 25 & 35 & 40 & 10 & 110 \\
\hline $\begin{array}{l}\text { Average Turnover ( } \mathrm{R} \\
\text { millions) }\end{array}$ & 12 & 53 & 718 & - & 309 \\
\hline $\begin{array}{l}\text { Average number of } \\
\text { employees }\end{array}$ & 79 & 258 & 1658 & $2036^{1)}$ & 870 \\
\hline $\begin{array}{l}\text { Capital expenditure - last } \\
\text { year }^{2)}\end{array}$ & $6 \%$ & $5 \%$ & $7 \%$ & - & $8 \%$ \\
\hline $\begin{array}{l}\text { Capital expenditure - last } \\
\text { three years }{ }^{2)}\end{array}$ & $10 \%$ & $10 \%$ & $17 \%$ & - & $19 \%$ \\
\hline
\end{tabular}

Table 2: Techniques used to evaluate capital expenditure projects

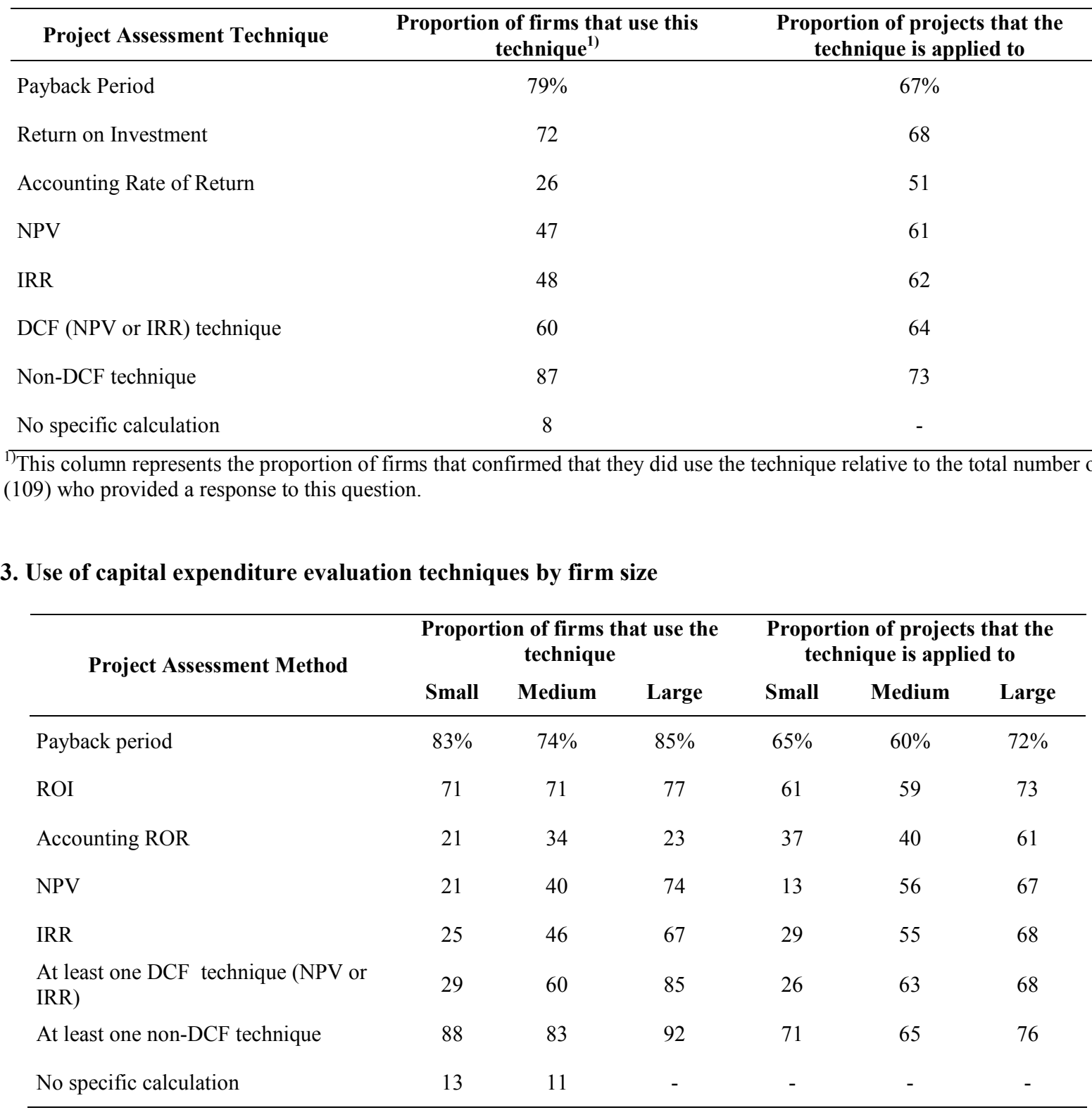


It is reported in Table 5 how firms adapt their DCF techniques to incorporate project specific risks. This data indicates that firms do respond to changing conditions - but generally do not use project specific risk adjusted discount rates in the way prescribed by corporate finance theory.

If viewed as a group, approximately 80 percent of the firms do not make an adjustment to the discount/hurdle rate for every separate project evaluated. In fact in the small firm category, more firms don't make any adjustments at all (thirteen percent) to these rates than those that make project specific adjustments (eight percent). Larger firms do tend to make changes to their discount rates most often. Of all the conditions that determine these adjustments, external changes are by far the most important.

\section{Discussion}

\section{Use of DCF (NPV and IRR) techniques}

1. The majority of firms use only the NPV technique when evaluating their capital investment projects (i.e. no other techniques are used at all).

Only 47 percent of the 110 firms that responded to this question use the NPV technique (see Table 2). Of these, only three firms us it as their sole evaluation technique (Table 4). South African manufacturing firms do not act in the way described above.

2 All firms use at least one of the DCF techniques (NPV and/or IRR) when evaluating their capital investment projects.

Only 55 percent of the firms surveyed use a DCF technique when evaluating their capital investment projects (Table 4). A significant minority of the respondents (45 percent) do not use these types of evaluation techniques at all. This proposition is not supported by the data.

3. Of the DCF techniques used, the NPV technique is used more often that the IRR technique.

On average, the IRR technique is used more often than the NPV technique (48 percent vs. 47 percent - see Table 2). This difference is relatively small and a case could be made for them being used on an equal basis. In any event, the data does not support this proposition.
4. The use of DCF techniques is positively related to firm size.

There is a clear increase in the average usage of both the NPV technique and DCF techniques as the firm size increases (see Table 3). However, a change in the size of the firm is not correlated with a decline in usage of non-DCF techniques. This indicates that larger firms are using DCF methods as part of a portfolio of evaluation techniques - and not on their own as prescribed by corporate finance theory.

\section{Use of DCF vs. non-DCF techniques}

5. The majority of firms do not use non-DCF techniques; 87 percent of the firms surveyed indicated that they use a non-DCF technique (see Table 2). This statement is thus not supported by the data.

6. Firms use DCF techniques more often than they use non-DCF techniques.

60 percent of the firms surveyed use DCF techniques compared to 87 percent that use non-DCF techniques (see Table 2). The use of DCF techniques is thus dominated by the use of other, non-DCF techniques.

\section{Use of combinations of techniques}

7. The firms that use DCF techniques use only these techniques (i.e. these firms do not use them in conjunction with other, non-DCF techniques).

Only five percent of the firms surveyed use a DCF technique only (see Table 4). 55 percent of the firms use a combination of DCF and non-DCF techniques. 34 percent of the firms only use non-DCF techniques. Firms thus are clearly using bundles of techniques when evaluating capital investment projects rather than a single DCF technique.

8. If a combination of DCF and non-DCF techniques is used, the DCF techniques are applied more often than the non-DCF techniques.

As is reported in Table 4, 55 percent of the firms surveyed (or 60 firms) use a combination of DCF and non-DCF techniques. To test which of these types of techniques are used more often, the relative use of DCF vs. non-DCF techniques within these 60 firms is presented in Table 6 . In each group, the firms apply the non-DCF techniques to more projects than the DCF techniques.

Table 4. Combination of techniques used to evaluate capital expenditure projects

\begin{tabular}{lcc}
\hline \multicolumn{1}{c}{ Evaluation Technique } & Result & Number of Responses \\
\hline Average number of techniques used & 3.0 & 100 \\
Firms use both NPV and IRR techniques & $35 \%$ & 38 \\
Only NPV used (no other techniques) & $3 \%$ & 3 \\
Only IRR used (no other techniques) & $1 \%$ & 1 \\
A DCF technique (either NPV and/or IRR) only & $5 \%$ & 5 \\
A non DCF technique (no NPV or IRR) only & $34 \%$ & 37
\end{tabular}


Table 5. Adjustments to the hurdle/discount rate used (all firms)

\begin{tabular}{lcccc}
\hline$\quad$ Adjustments made & All & Small & Medium & Large \\
\hline For each project & $21 \%$ & $8 \%$ & $23 \%$ & $28 \%$ \\
Changing external conditions & 40 & 25 & 29 & 67 \\
Changing internal conditions & 29 & 17 & 26 & 44 \\
On a regular basis & 6 & - & 9 & 10 \\
Not sure how/why & 5 & 4 & 11 & - \\
No changes made & 9 & 13 & 11 & 5 \\
\hline
\end{tabular}

Table 6. The extent to which DCF and Non-DCF techniques are applied to projects (when both are used by a firm)

\begin{tabular}{ccccc}
\hline Technique & All ${ }^{1)}$ & Small & Medium & Large \\
\hline DCF (NPV or IRR) & $61 \%$ & $26 \%$ & $59 \%$ & $66 \%$ \\
Non-DCF & 72 & 61 & 67 & 76 \\
${ }^{\mathrm{T}}$ This includes four firms which did not report their turnover and thus which could not be allocated to a size group.
\end{tabular}

\section{Adjusting for risk}

9. The majority of firms adjust the discount rate used in their NPV calculations to incorporate the project specific risk.

Only 21 percent of firms do adjust their discount rates (or hurdle rates if using IRR) to account for project specific risk (see Table 5). Again, this proposition is not supported by the data.

In summary, the data collected for South African manufacturing firm suggests that these firms do not act in accordance with the prescriptions of corporate finance theory. They do not use NPV techniques in isolation with project specific risk adjusted discount rates when evaluating capital investment projects. 45 percent of them do not use NPV techniques at all and when they do use them, they are used in conjunction with non-DCF techniques. The nonDCF techniques are both used by more firms and are applied more often when both types of methods are used.

The presence of these consistent deviations from the prescriptions of corporate finance theory may be due to managers' lack of experience (or knowledge) of these methods. The fact that more firms are using DCF methods more now than in the past would support this hypothesis ${ }^{7}$. However, the fact that these methods are being added to, rather than replacing, other non-DCF methods suggests that something is missing from the body of the theory of capital

\footnotetext{
${ }^{7)}$ See Andrews and Butler (1986), Pike (1996) and Klammer and Walker (1984) for evidence of this in South Africa, the United Kingdom and the United States respectively.
}

budgeting - something that managers attempt to deal with by using a portfolio of techniques.

One hypothesis is that this behaviour may be due to the presence of risk (or uncertainty) in the decision making process. DCF techniques' strength as a decision making support depends directly on the accuracy (or confidence) of the decision maker's inputs to the model i.e. their ability to accurately forecast the future. Should this confidence be lacking, alternative techniques may help make the decision. For example, the PP method provides decision makers with an indication of how exposed their capital investment is in a particular project. Its continued popularity suggests that it provides decision makers with a way of dealing with perceived risk in a way that the DCF technique does not. In any event, the evidence of the use of multiple methods suggests that no one of them provides a sufficient basis for managers to make the decision.

\section{Conclusion}

Evidence of three systematic deviations from the behaviour prescribed by corporate finance theory was tested for manufacturing firms in South Africa through the use of a postal questionnaire. Firstly, a significant proportion of firms do not use DCF techniques at all to evaluate capital expenditure decisions. Secondly, when they do use DCF techniques, they are used in conjunction with other, theoretically inferior evaluation techniques. Finally, project specific discount rates (reflecting the unique risk of the project's expected cash flows) are not used by the majority of firms. The evidence from this study suggests that all of these observations are accurate for the firms in this sector. 
The results presented in this paper indicate the existence and extent of this behaviour, but are not sufficient to explain it. Additional evidence regarding the capital expenditure process itself is required in order to answer these questions. A necessary focus of this future work should be on the rationale for the use of a combination of techniques, and in particular, what help each method used provides to decision makers when making their capital investment decisions.

\section{References}

Andrews, G.S. \& Butler, F. 1986. 'Criteria for major investment decisions', The Investment Analysts Journal, 27: 31-37.

Copeland, T.E. \& Weston, J.F. 1992. Financial theory and corporate policy. New York: Addison-Wesley Publishing Company Inc.

Dillman, D.A. 1978. Mail and telephone surveys: The total design method. New York: John Wiley \& Sons, Inc.

Hall, J.H. 2000. 'Investigating aspects of the capital budgeting process used in the evaluation of investment projects', South African Journal of Economic and Management Studies, 3(3): 354-368.

Hall, J.H. 2001. 'Risk analysis and evaluation of capital investment projects', South African Journal of Economic and Management Studies, 4(2): 398-411.

Klammer, T.P. \& Walker, M.C. 1984. 'The continuing increase in the use of sophisticated capital budgeting techniques', California Management Review, 27(Fall): 137148.

Nickell, S.J. 1978. The investment decisions of firms. Cambridge: Cambridge University Press.

Parry, H.M.A. \& Firer, C. 1990. 'Capital budgeting under uncertainty: an empirical study', South African Journal of Business Management, 21(3): 52-58.

Pike, R.H. 1996. 'A longitudinal survey on capital budgeting practices', Journal of Business, Finance and Accounting, 23(1): 79-92.

\section{Acknowledgements}

The author would like to thank Jonathan Michie and Colin Firer for their comments on this paper. He would also like to thank the South African Chamber of Commerce for their support of this research effort. 